\title{
Promotion of in vivo degradability, vascularization and osteogenesis of calcium sulfate-based bone cements containing nanoporous lithium doping magnesium silicate
}

\author{
This article was published in the following Dove Press journal: \\ International Journal of Nanomedicine \\ 17 February 2017 \\ Number of times this article has been viewed
}

\author{
Liehu Cao',* \\ Weizong Wengl,* \\ Xiao Chen ${ }^{1, *}$ \\ Jun Zhang' \\ Qirong Zhou' \\ Jin Cui' \\ Yuechao Zhao' \\ Jung-Woog Shin² \\ Jiacan Su'
}

'Department of Orthopaedics Trauma, Changhai Hospital, Second Military

Medical University, Shanghai, People's

Republic of China; ${ }^{2}$ Department

of Biomedical Engineering, Inje

University, Gimhae, Republic of Korea

*These authors contributed equally to this work
Correspondence: Jiacan Su

Department of Orthopaedics Trauma,

Changhai Hospital, Second Military

Medical University, Shanghai 200433,

People's Republic of China

Tel +86 2I 81873400

Fax +86 21 81873398

Email drsujiacan@।63.com

\begin{abstract}
Nanoporous lithium doping magnesium silicate (nl-MS) was introduced into calcium sulfate hemihydrate to prepare calcium sulfate composite (nl-MSC) bone cements. The introduction of nl-MS improved the in vitro degradability of nl-MSC cements, which could neutralize acidic degradable products of calcium sulfate and prevented the $\mathrm{pH}$ from dropping. The cements were implanted into the bone defects of femur bone of rabbits, and the results of histological and immunohistochemical analysis revealed that massive new bone tissue formed in the defects while the cements were degradable, indicating that the osteogenesis and degradability of the nl-MSC cements were much better than the control calcium sulfate dihydrate (CSD) cements. Furthermore, the positive expression of vascular endothelial growth factor and collagen type I for nl-MSC cements was higher than CSD, indicating that addition of nl-MS into the cements enhanced vascularization and osteogenic differentiation. The results suggested that the nl-MSC cements with good biocompatibility and degradability could promote vascularization and osteogenesis, and had great potential to treat bone defects.
\end{abstract}

Keywords: bone cements, nanoporous lithium doping magnesium silicate, degradability, vascularization, osteogenesis

\section{Introduction}

Calcium sulfate-based bone cement has a long history as the bone-repair biomaterial for clinical application due to its good degradability and biocompatibility. ${ }^{1}$ Calcium sulfate hemihydrate $(\mathrm{CSH})$ can react with water immediately and transform into calcium sulfate dihydrate (CSD). ${ }^{2}$ However, CSD cement has some drawbacks that significantly limit its clinical application, such as short setting time, low bioactivity and poor ability to form new bone tissue. ${ }^{3,4}$ Additionally, the degradation of CSD in vivo causes local acidity, which can lead to inflammatory reaction in host tissues. ${ }^{5}$

Nanoporous silicates (n-SCs) as new kinds of biomaterials have high specific surface area and pore volume, which may greatly accelerate the kinetics of apatite deposition, thus enhancing the bone-forming bioactivity. ${ }^{6}$ Furthermore, the degradable products of n-SCs present weak alkalinity, which is favorable for cell growth and tissue regeneration. ${ }^{6,7}$ Silicate-based biomaterials have been reported to enhance vascularization of osteoblastic cells through stimulating the expression of vascular endothelial growth factor (VEGF) from osteoblastic cells or neighbor cells, and it has been widely accepted that angiogenesis could enhance bone formation. ${ }^{8-10}$ A previous 
study has shown that silicate biomaterials are able to stimulate osteogenic differentiation of osteoblastic cells, and subsequently, strongly affect new bone formation..$^{9,11}$

Lithium ( $\mathrm{Li}$ ) is an important trace element in an organism, and previous studies have shown that Li ions stimulated the proliferation and osteogenic differentiation of bone marrow mesenchymal stem cells (BMSCs) through activation of the Wnt signaling pathway in vitro, and oral administration of Li ions enhanced subchondral bone formation via activation of the Wnt signaling pathway. ${ }^{12,13}$ Moreover, Li-containing mesoporous bioglass scaffolds promoted renascence of subchondral bone and enhanced hyaline cartilage tissue restoration in rabbit models of osteochondral defects. ${ }^{14}$ In this study, nanoporous lithium doping magnesium silicate (nl-MS) was introduced into $\mathrm{CSH}$ to prepare calcium sulfate-based composite (nl-MSC) cements, and the in vivo degradability, vascularization and osteogenesis of nl-MSC cements were investigated.

\section{Materials and methods Preparation of $\mathrm{nl}-\mathrm{MS}$ and $\mathrm{nl}-\mathrm{MSC}$ cements}

The nl-MS was synthesized using a sol-gel method. Briefly, tetraethyl orthosilicate $(3 \mathrm{~mL})$ was dissolved into ethanol $(25 \mathrm{~mL})$ to form solution A. The cetyltrimethylammoniumchloride (CTAC, $2 \mathrm{~g}$ ) and ammonium hydroxide (28 wt\%, $4.5 \mathrm{~mL})$ were dissolved into ethanol $(50 \mathrm{~mL})$ to form solution B. Lithium chloride $(0.25 \mathrm{~g})$ was dissolved into ethanol $(10 \mathrm{~mL})$ to form solution $\mathrm{C}$. Then, solutions A and B were mixed together under rapid agitation, and solution $\mathrm{C}(1 \mathrm{~mL})$ was doped into mixed solution drop by drop. Then, the mixed solution was stirred vigorously for another $3 \mathrm{~h}$, and the products collected were centrifuged, and then washed 3 times with water and ethanol, respectively. The final products of nl-MS were dried for $24 \mathrm{~h}$ at room temperature, and then calcinated at $600^{\circ} \mathrm{C}$ for $4 \mathrm{~h}$ in air to remove CTAC. The nl-MS was characterized by high-resolution transmission electron microscopy (JEM-2010; JEOL, Tokyo, Japan).

$\mathrm{CSH}$ powders were prepared by heating CSD $\left(\mathrm{CaSO}_{4} \cdot 2 \mathrm{H}_{2} \mathrm{O}\right)$ in a muffle furnace $(\mathrm{SX} 2-2.5-12 \mathrm{~N}$; Yiheng, Shanghai, People's Republic of China) at $150^{\circ} \mathrm{C}$ for $12 \mathrm{~h}$ and then at $120^{\circ} \mathrm{C}$ for $6 \mathrm{~h}$. The CSD, $10 \mathrm{nl}-\mathrm{MSC}$ and $20 \mathrm{nl}-\mathrm{MSC}$ cement powders were prepared by mixing $\mathrm{CSH}$ powders with 0,10 and $20 \mathrm{wt} \% \mathrm{nl}-\mathrm{MS}$ powders. The CSD, $10 \mathrm{nl}-\mathrm{MSC}$ and 20 nl-MSC cement pastes were prepared by mixing cement powders with deionized water (a ratio of cement powder to liquid of 1:0.8 g/mL). The as-prepared cement pastes were cast into Teflon molds under a pressure of $2 \mathrm{MPa}$ for $1 \mathrm{~min}$. The samples $(\Phi 10 \times 2 \mathrm{~mm}$ and $\Phi 6 \times 6 \mathrm{~mm})$ were taken out and then cured under the condition of $100 \%$ air humidity and $37^{\circ} \mathrm{C}$ for 2 days. After the hardening of cements, the composition and microstructure of the CSD, 10 nl-MSC and 20 nl-MSC cements were characterized by X-ray diffractometry (XRD; Geigerflex; Rigaku Co. Ltd., Tokyo, Japan), Fourier transform infrared spectroscopy (Thermo Nicolet 6700; Thermo Fisher Scientific, Waltham, MA, USA) and scanning electron microscopy (SEM; S-3400N; Hitachi, Tokyo, Japan), respectively.

\section{Degradation of cements in phosphate- buffered saline}

Samples $(\Phi 10 \times 2 \mathrm{~mm})$ of CSD, $10 \mathrm{nl}-\mathrm{MSC}$ and $20 \mathrm{nl}-\mathrm{MSC}$ cements were immersed in phosphate-buffered saline (PBS, $\mathrm{pH} 7.4$ ) at $37^{\circ} \mathrm{C}$ with a solid/liquid ratio of $0.1 \mathrm{~g} / 20 \mathrm{~mL}$, and the solution was refreshed every 7 days. At different time points, the specimens were removed from the liquid, rinsed with deionized water and dried in an oven at $90^{\circ} \mathrm{C}$ for $8 \mathrm{~h}$. The percentage of weight loss was calculated as the following formula: weight loss $(\%)=\left(W_{0}-W_{\mathrm{t}}\right) / W_{0} \times 100$, where $W_{0}$ is the starting dry weight and $W_{\mathrm{t}}$ is the dry weight at time $\mathrm{t}$. The change of $\mathrm{pH}$ values of the solutions after the cements were soaked for different times was also determined with a pH meter (PHS-2C; JingkeLeici, Shanghai, People's Republic of China).

\section{Implantation of cements into bone defects of rabbits}

The in vivo experiments were performed according to the NIH guidelines for the care and use of laboratory animals (NIH Publication No 85-23 Rev 1985) and were approved by the ethical committee of the Shanghai University of Traditional Chinese Medicine. The 24 New Zealand White rabbits (female, about $2.1 \mathrm{~kg}$ ) were divided into 3 groups. The rabbits were anesthetized with pentobarbital sodium. The incision sites were made on the right femur of rabbits, and then $\Phi 6 \times 6 \mathrm{~mm}$ defects were made in the thighbone of the rabbits. The CSD, 10 nl-MSC and 20 nl-MSC cements were implanted into the defects. At 4, 8 and 12 weeks after surgery, the rabbits were sacrificed, and the thighbones were taken out and placed into 4\% neutral-buffered formalin. All the rabbits were raised, operated and handled in the Research Center for Laboratory Animals of the Shanghai University of Traditional Chinese Medicine.

\section{SRmCT images analysis}

The synchrotron radiation micro-computed tomography (SRmCT) measurement was performed at beamline BL13W of the Shanghai Synchrotron Radiation Facility (Shanghai, 
People's Republic of China) using a monochromatic beam with an energy of $30 \mathrm{keV}$ and a distance (sample-to-detector) of $1.6 \mathrm{~m}$. The pixel size was set to $14 \times 14 \mu \mathrm{m}$, and a spacing of $14 \mu \mathrm{m}$ was used to obtain a continuous flat image with a resolution of $1,024 \times 1,024$. The images were reconstructed by supporting software (VGStudio MAX 2.1) to generate 3-dimensional (3D) grayscale images.

\section{Histological evaluation}

The specimens were decalcified in 5\% formic acid (SigmaAldrich, St Louis, MO, USA) at $4^{\circ} \mathrm{C}$ for 15 days, and then dehydrated in a gradient ethanol series and embedded in paraffin. After that, the specimens were cut into $20-\mu \mathrm{m}-$ thick sections with a sawing microtome (Leica 1600; Leica Microsystems, Wetzlar, Germany). All sections were stained with hematoxylin/eosin (H\&E). The newly formed bone was observed under a light microscope (TE2000U; Nikon, Tokyo, Japan). The ratio of newly formed bone and residual material was calculated by a semiautomatic image analysis system (Image Pro Plus 6.0).

\section{Immunohistochemical evaluation}

The immunohistochemical labeling of the decalcified sections was carried out using the immunoperoxidase detection method with the primary antibodies anti-VEGF and antiCOL-I (1:50 dilution; Abcam, Inc., Cambridge, UK) for $12 \mathrm{~h}$ at $4{ }^{\circ} \mathrm{C}$. Then, the sections were washed 3 times with PBS. After that, the anti-IgG biotinylated antibody (Abcam, Inc.) at a 1:200 concentration was incubated as secondary antibody for $30 \mathrm{~min}$ at $37^{\circ} \mathrm{C}$. Thereafter, the sections were incubated with horseradish peroxidase-conjugated avidin-biotin complex (Abcam, Inc.) for another $15 \mathrm{~min}$, followed by buffered 3,3'-diaminobenzidinetetrahydrochloride (Abcam, Inc.) as chromogen. The slides were then observed under a light microscope (TE2000U; Nikon). The positive expression ratio was calculated by a semiautomatic image analysis system (Image Pro Plus 6.0).

\section{Statistical analysis}

All quantitative data expressed as the mean \pm standard deviation were analyzed with ORIGIN v 8.0 (Originlab Corporation, Northampton, MA, USA). Statistical analysis was performed with one-way analysis of variance. A value of $P<0.05$ was considered statistically significant.

\section{Results TEM and XRD analysis}

Figure 1 reveals the TEM image of nl-MS, and XRD of CSD, $10 \mathrm{nl}-\mathrm{MSC}$ and $20 \mathrm{nl}$-MSC cements. It can be seen that the nl-MS exhibited sphere-like particles with a size of about $100 \mathrm{~nm}$. In addition, it can be seen from XRD that the diffraction peak of CSD appeared at $2 \theta=11.7^{\circ}, 20.8^{\circ}, 23.4^{\circ}, 29.2^{\circ}, 31.2^{\circ}$ and $33.4^{\circ}$, indicating that the $\mathrm{CSH}$ had transformed into CSD. Correspondingly, the peak of CSD was found in $10 \mathrm{nl}-\mathrm{MSC}$ and 20 nl-MSC cements, but the peak intensity of CSD significantly decreased with the increase of nl-MS content.

\section{Infrared analysis}

Figure 2 shows the infrared spectrum (IR) of nl-MS, CSD, 10 nl-MSC and 20 nl-MSC. The peaks at 3,579, 3,493 and $1,658 \mathrm{~cm}^{-1}$ were typical of $\mathrm{H}_{2} \mathrm{O}$ or $\mathrm{OH}^{-}$, while the absorption band at $802 \mathrm{~cm}^{-1}$ was attributed to the $\mathrm{Si}=\mathrm{O}$ stretching vibration, and the peak at $1,176 \mathrm{~cm}^{-1}$ was attributed to $\mathrm{SO}_{4}{ }^{2-}$ stretching vibration. All the characteristic peaks of CSD can be found in the $10 \mathrm{nl}-\mathrm{MSC}$ and $20 \mathrm{nl}-\mathrm{MSC}$ cements, and no obvious change of the characteristic peaks of CSD was found.
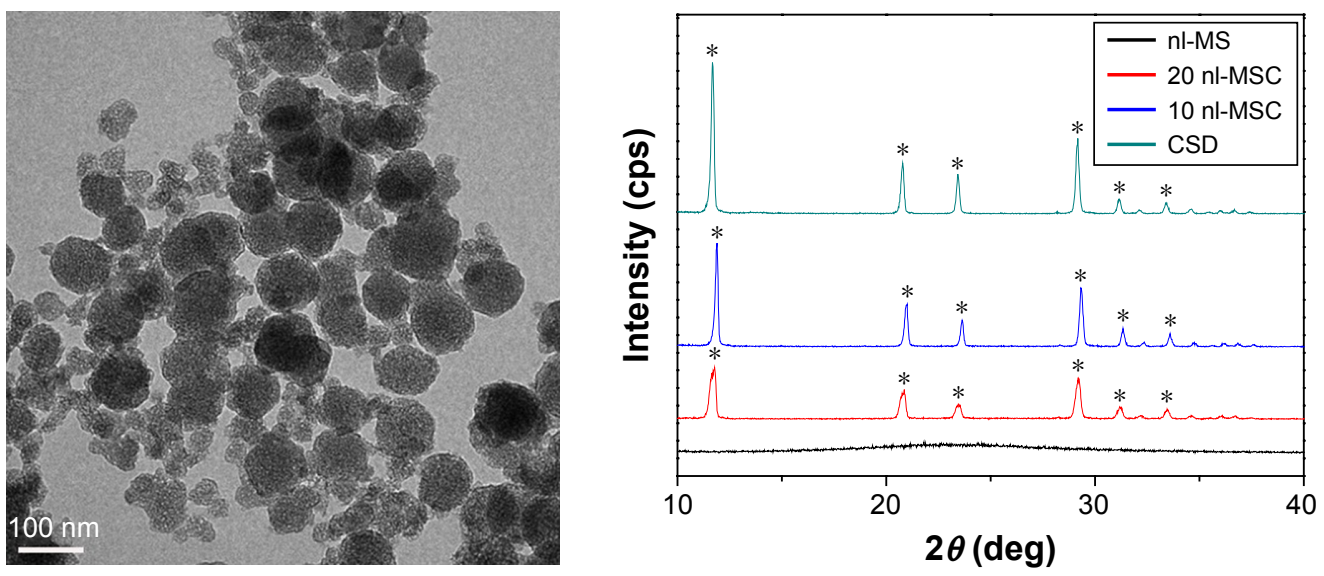

Figure I TEM image of nl-MS, and XRD of CSD, 10 nl-MSC and 20 nl-MSC cements. Asterisks represent the peaks of CSD. Abbreviations: TEM, transmission electron microscopy; nl-MS, nanoporous lithium doping magnesium silicate; XRD, X-ray diffractometry; CSD, calcium sulfate dihydrate; nl-MSC, nl-MS composite. 

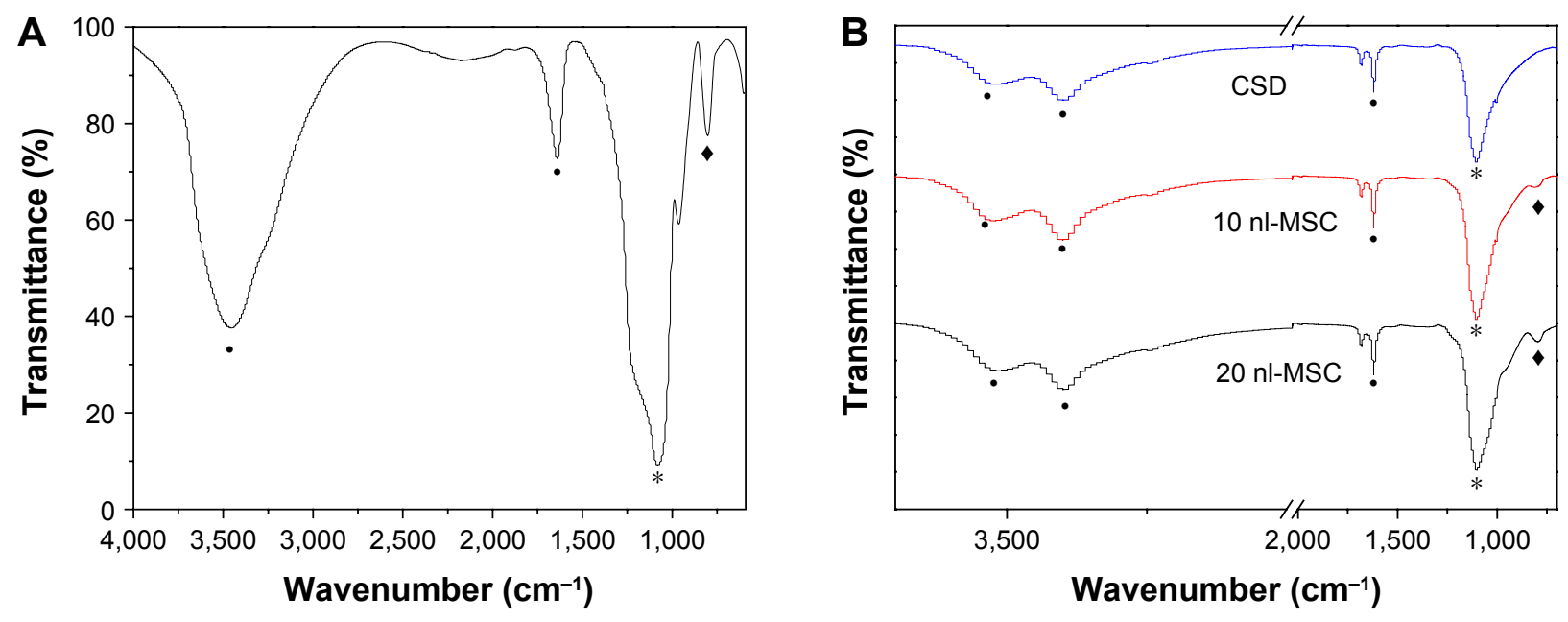

Figure 2 IR of nl-MS (A), CSD, 10 nl-MSC and 20 nl-MSC (B) cements. Filled circles represent $\mathrm{H}_{2} \mathrm{O}_{\text {or }} \mathrm{OH}^{-}$, filled diamonds represent $\mathrm{Si}=\mathrm{O}$ and asterisks represent $\mathrm{SO}_{4}{ }^{2-}$. Note: (B) FTIR of CSD, $10 \mathrm{nl}-M S C$ and 20 nl-MSC.

Abbreviations: IR, infrared; nl-MS, nanoporous lithium doping magnesium silicate; CSD, calcium sulfate dihydrate; nl-MSC, nl-MS composite; IR, infrared spectrum.

\section{SEM analysis of cements}

The SEM images of surface morphology of the cements are shown in Figure 3. The low-magnification images show that the surface of the CSD cement was compact (Figure 3A), indicating that the $\mathrm{CSH}\left(\mathrm{CaSO}_{4} \cdot 1 / 2 \mathrm{H}_{2} \mathrm{O}\right)$ had transformed into $\mathrm{CSD}\left(\mathrm{CaSO}_{4} \cdot 2 \mathrm{H}_{2} \mathrm{O}\right)$. However, the surfaces of the cements were loose (Figure $3 \mathrm{C}$ and $\mathrm{E}$ ), and some nl-MSs were observed on the surfaces of $10 \mathrm{nl}-\mathrm{MSC}$ and $20 \mathrm{nl}-\mathrm{MSC}$ cements. At high magnification, the rod-like microcrystalline CSD was seen (Figure 3B), while microporous CSD appeared on the surfaces of the $10 \mathrm{nl}-\mathrm{MSC}$ and $20 \mathrm{nl}-\mathrm{MSC}$ cements, showing that the amorphous nl-MS dispersed in the microcrystalline CSD (Figure 3D and F).

\section{Degradation of cements in PBS}

Figure 4A shows the weight loss of the CSD, 10 nl-MSC and $20 \mathrm{nl}$-MSC cements after being soaked into PBS for different times. The weight loss of the cements increased with time. Moreover, the weight loss of the cements increased with the increase of nl-MS content. At 12 weeks, the weight loss ratio of the CSD, $10 \mathrm{nl}-\mathrm{MSC}$ and $20 \mathrm{nl}-\mathrm{MSC}$ cements was $55.4,64.4$ and $72.8 \mathrm{wt} \%$, respectively. Figure 4B shows the $\mathrm{pH}$ changes of the solutions after immersion of specimens in PBS for different periods of time. The $\mathrm{pH}$ of the solution for CSD significantly decreased at the first 2 weeks (from 7.40 to 7.15); after that, it began to slowly decline and dropped to 6.86 at 12 weeks. However, the $\mathrm{pH}$ of the solution for $10 \mathrm{nl}-\mathrm{MSC}$ and $20 \mathrm{nl}-\mathrm{MSC}$ cements dropped from 7.40 to 7.18 and 7.27 at the first 2 weeks; after that, the $\mathrm{pH}$ value began to increase, and reached 7.38 and 7.45 at 12 weeks, respectively.

\section{SRmCT image analysis}

Figure 5 shows the cross-section of the 3D reconstruction images of SRmCT after CSD, 10 nl-MSC and 20 nl-MSC cements were implanted into bone defects of rabbit femur for different periods of time. From these images, it can be seen that the bone defects were gradually repaired with time.

\section{Histological evaluation}

Figure 6 shows the histological sections of H\&E staining of CSD, $10 \mathrm{nl}$-MSC and $20 \mathrm{nl}-\mathrm{MSC}$ cement after being implanted in vivo for 4,8 and 12 weeks. The amounts of new bone tissue for all the cements increased with time. In addition, the amounts of new bone tissue increased with the increase of nl-MS content (CSD $<10$ nl-MSC $<20$ nl-MSC). Meanwhile, it was found that the cements in the bone defects gradually reduced with time, indicating the gradual degradation of the cements.

Figure 7A shows the quantitative analysis of new bone formation after the cements were implanted in vivo for different times. It was found that the percentage of new bone formation increased with time for all the cements, and the percentage of new bone formation increased with the increase of nl-MS content (CSD $<10$ nl-MSC $<20$ nl-MSC). At 12 weeks, the percentages of new bone formation for CSD, $10 \mathrm{nl}-\mathrm{MSC}$ and $20 \mathrm{nl}-\mathrm{MSC}$ were $47.91 \%, 56.52 \%$ and $67.50 \%$, respectively.

Figure 7B shows the quantitative analysis of residual material ratio after the cements were implanted in vivo for different times. The residual material ratio decreased with time for all the cements, and the residual material ratio decreased with the increase of nl-MS content (CSD >10 nl-MSC $>20$ nl-MSC). 

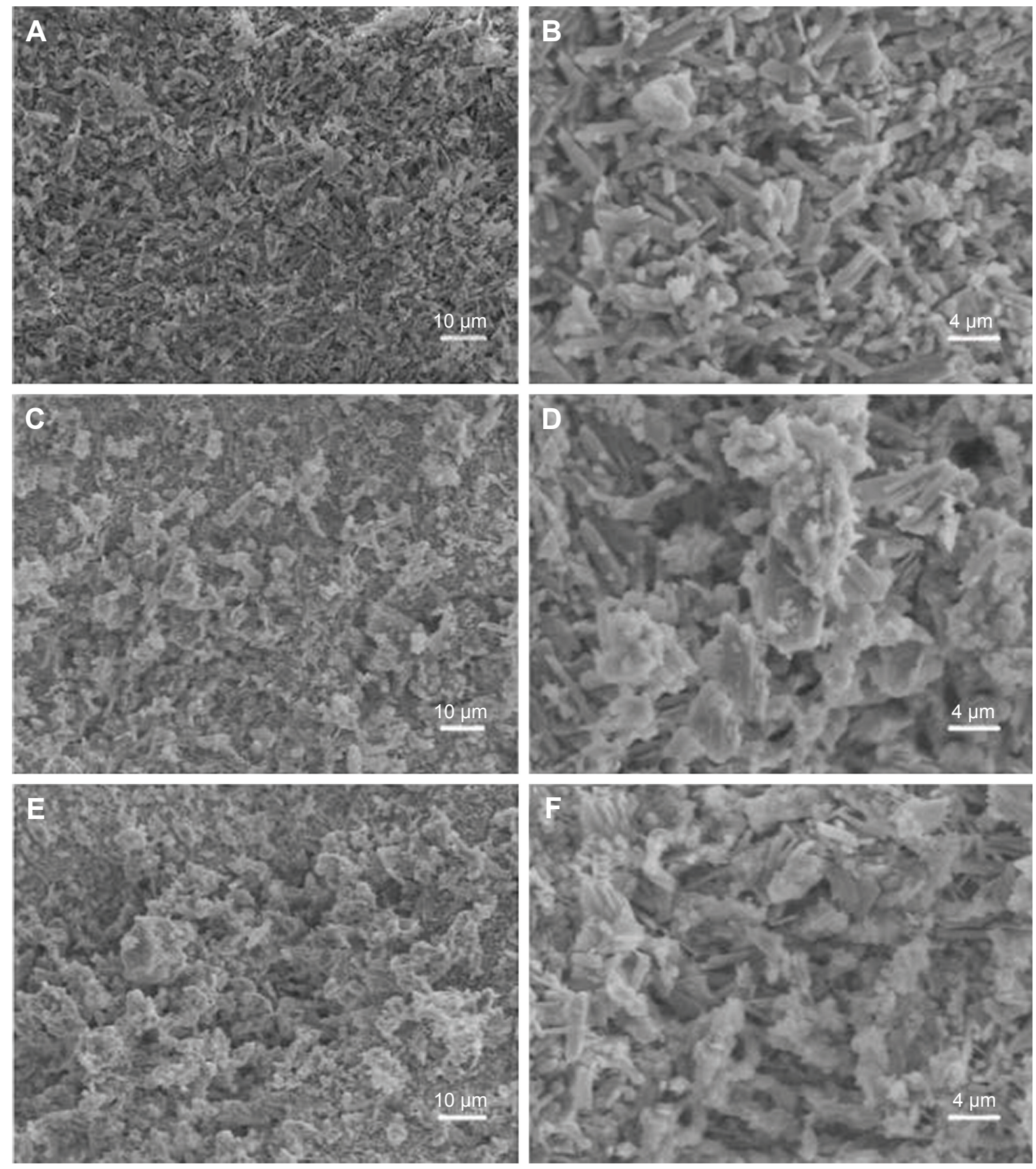

Figure 3 SEM images of surface morphology of CSD (A and B), $10 \mathrm{nl}-\mathrm{MSC}$ (C and $\mathbf{D})$ and $20 \mathrm{nl}-\mathrm{MSC}$ (E and $\mathbf{F})$ cements.

Abbreviations: SEM, scanning electron microscopy; CSD, calcium sulfate dihydrate; nl-MSC, nanoporous lithium doping magnesium silicate composite.

At 12 weeks, the percentages of residual materials for CSD, $10 \mathrm{nl}-\mathrm{MSC}$ and $20 \mathrm{nl}-\mathrm{MSC}$ were $12.20 \%, 9.43 \%$ and $6.34 \%$, respectively.

\section{Immunohistochemical analysis}

Figure 8 shows the immunohistological staining of VEGF after the cements were implanted in vivo for different times. It was found that the VEGF staining intensity for all the cements increased with time, and the VEGF staining intensity increased with the increase of nl-MS content. Figure 9 shows the quantitative analysis of the immunohistological positive expression ratio of VEGF after the cements were implanted in vivo for different times. It was found that the positive expression ratio for all the cements increased with time and nl-MS content. At 12 weeks, the positive expression ratios of VEGF for CSD, $10 \mathrm{nl}$-MSC and $20 \mathrm{nl}$-MSC were $26.97 \%$, $33.56 \%$ and $38.79 \%$, respectively.

Figure 10 shows the immunohistological staining of collagen type I (COL-I) by histological sections after the cements were implanted in vivo for different times. It was found that the COL-I staining intensity for all the cements increased with time, and the COL-I staining intensity increased with the 

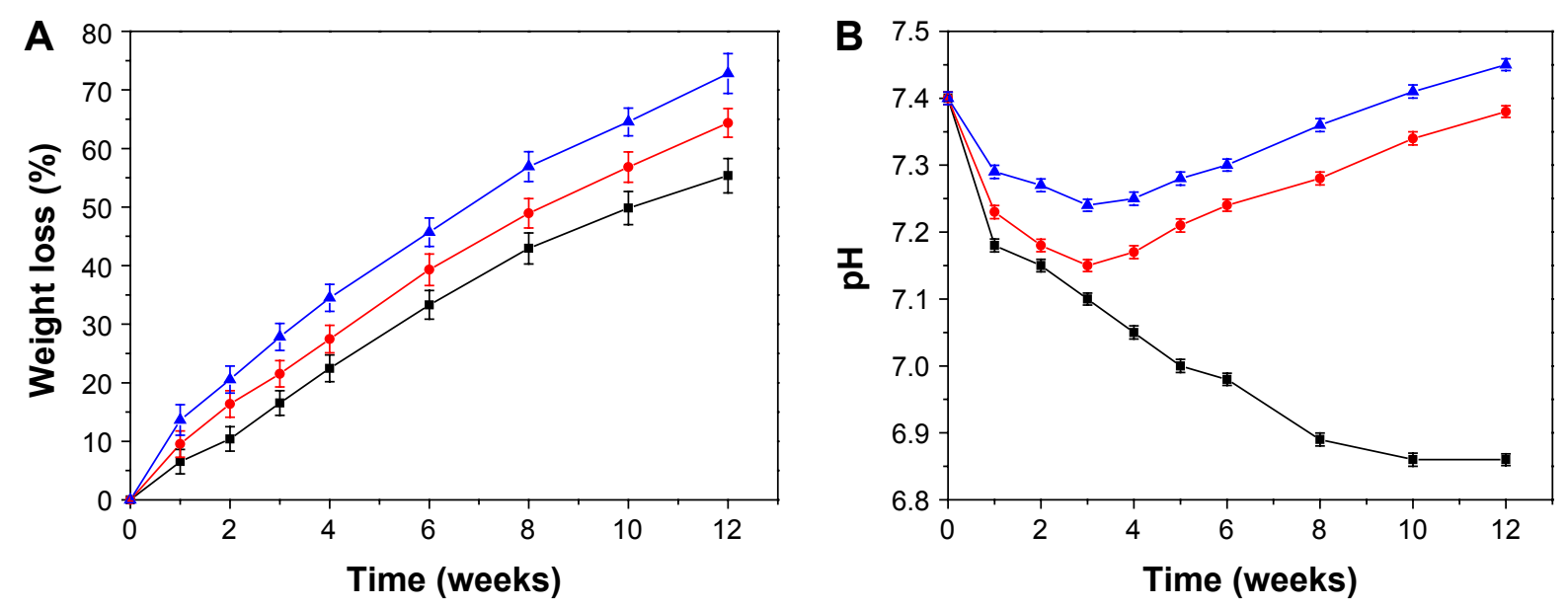

$$
\longrightarrow \text { CSD } \multimap 10 \mathrm{nl}-\mathrm{MSC} \multimap 20 \mathrm{nl}-\mathrm{MSC}
$$

Figure 4 Weight loss (A) of the cements in the PBS and change of $\mathrm{pH}(\mathbf{B})$ of solution after the cements were soaked for different times. Abbreviations: PBS, phosphate-buffered saline; CSD, calcium sulfate dihydrate; nI-MSC, nanoporous lithium doping magnesium silicate composite.
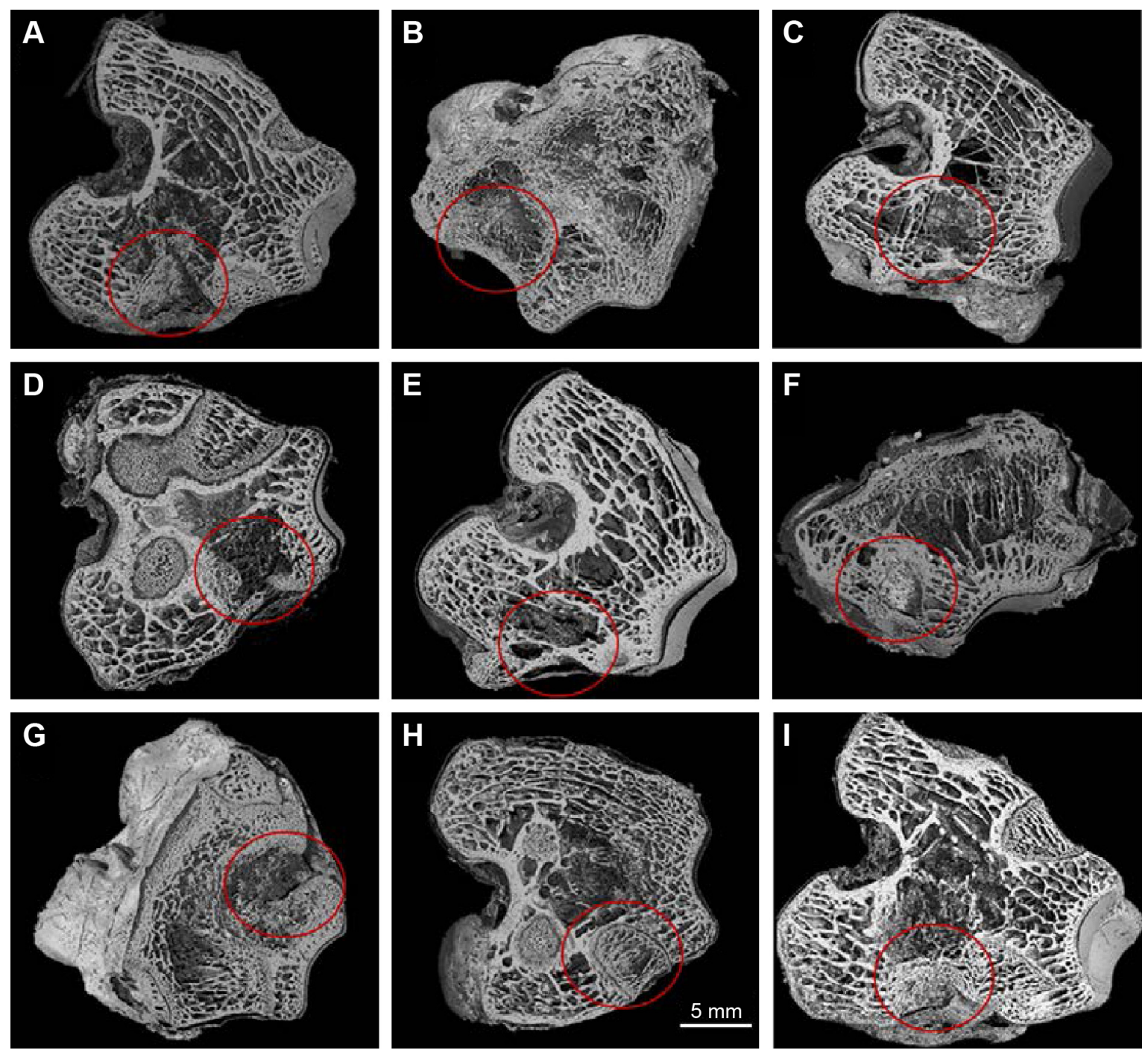

Figure 5 Three-dimensional reconstruction images (cross-section) of SRmCT after CSD (A-C), 10 nl-MSC (D-F) and 20 nl-MSC (G-I) were implanted into bone defects of rabbit femur for 4 (A, D and $\mathbf{G}), 8$ (B, E and $\mathbf{H})$ and 12 (C, $\mathbf{F}$ and $\mathbf{I})$ weeks. Bone defects are marked with red circles.

Abbreviations: SRmCT, synchrotron radiation micro-computed tomography; CSD, calcium sulfate dihydrate; nl-MSC, nanoporous lithium doping magnesium silicate composite. 

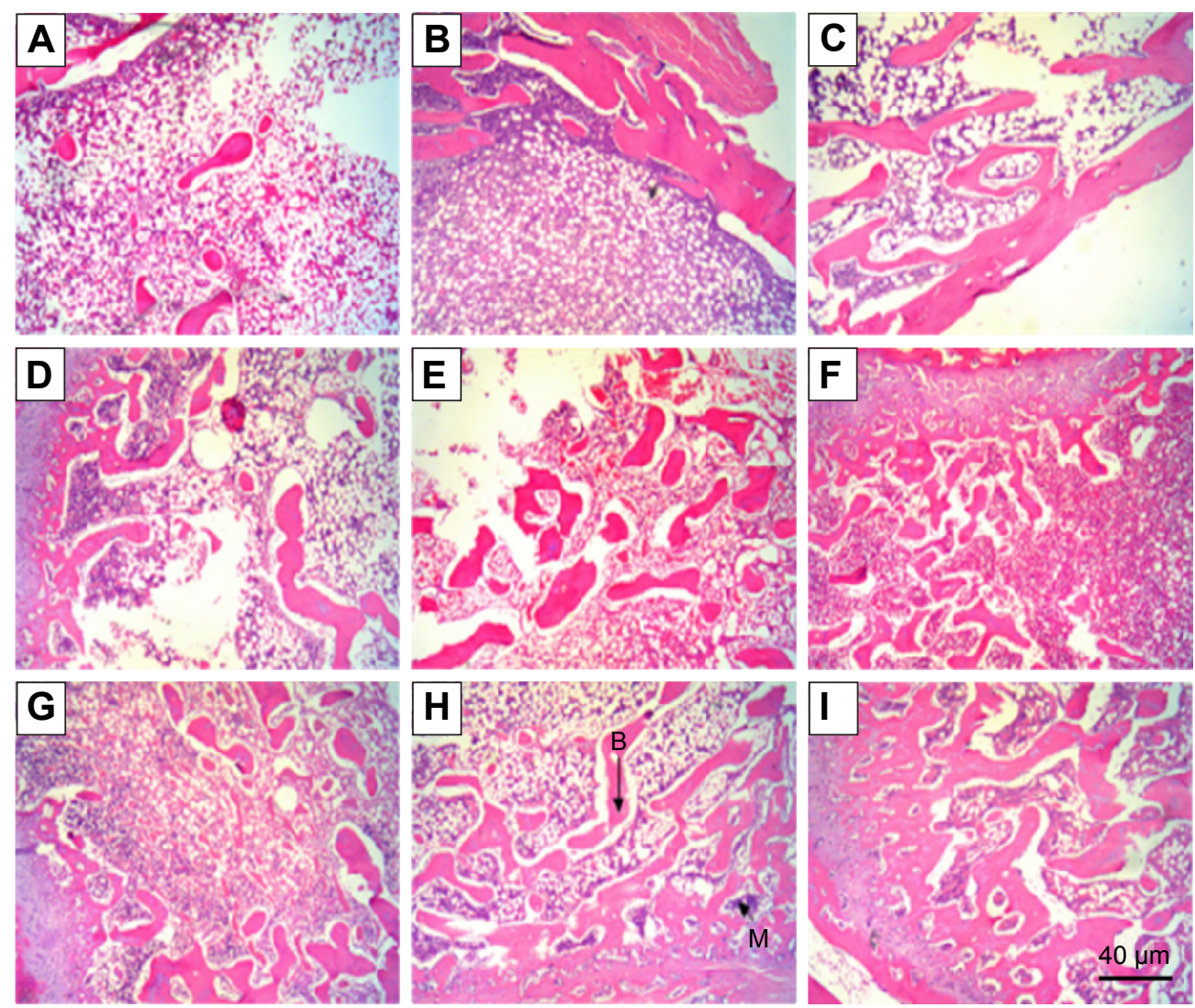

Figure 6 Histological sections of H\&E staining after CSH (A-C; 40x), 10 nl-MSC (D-F; 40x) and 20 nl-MSC (G-I; 40x) were implanted in vivo for 4 (A, D and G), 8 (B, E and $\mathbf{H})$ and 12 (C, $\mathbf{F}$ and $\mathbf{I})$ weeks (B: new bone and $\mathrm{M}$ : materials).

Abbreviations: $\mathrm{H \& E}$, hematoxylin/eosin; $\mathrm{CSH}$, calcium sulfate hemihydrate; nl-MSC, nanoporous lithium doping magnesium silicate composite.

increase of nl-MS content. Figure 11 shows the quantitative analysis of the immunohistological positive expression ratio of COL-I after the cements were implanted in vivo for different times. It was found that the positive expression ratio for all the cements increased with time and nl-MS content. At 12 weeks, the positive expression ratios of COL-I for CSD, $10 \mathrm{nl}-\mathrm{MSC}$ and $20 \mathrm{nl}-\mathrm{MSC}$ cements were $22.70 \%, 30.34 \%$ and $48.30 \%$, respectively.
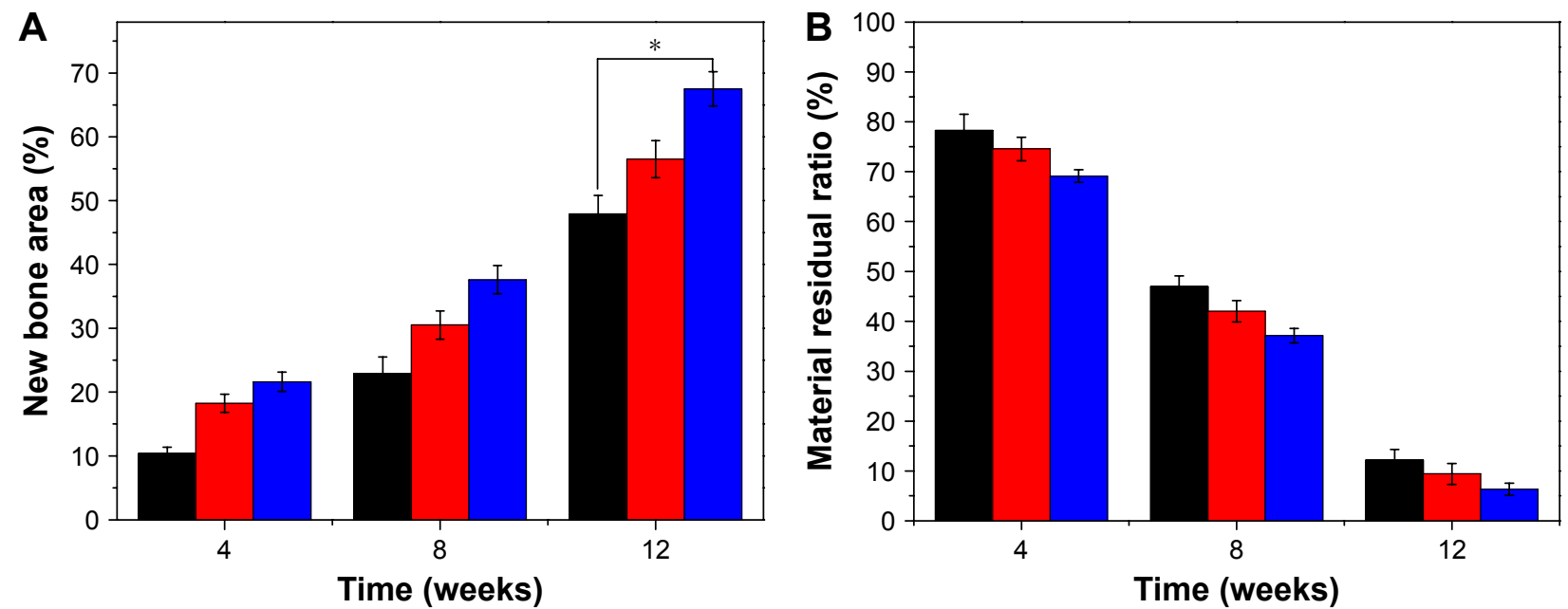

CSD

$10 \mathrm{nl}-\mathrm{MSC}$

$20 \mathrm{nl}-\mathrm{MSC}$

Figure 7 Quantitative comparison of the percentage of new bone formation $(\mathbf{A})$ and material residual ratio (B) by histological section after the implantation of cements in vivo for 4,8 and 12 weeks. Asterisk represents statistical significance $(P<0.05)$.

Abbreviations: CSD, calcium sulfate dihydrate; nl-MSC, nanoporous lithium doping magnesium silicate composite. 


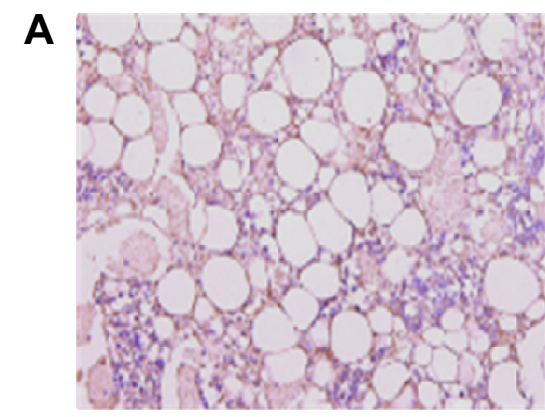

B
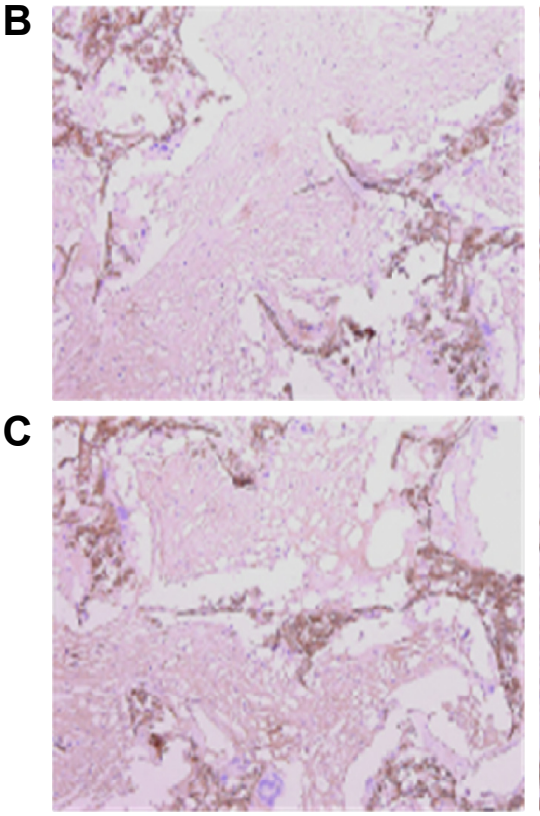

4 weeks
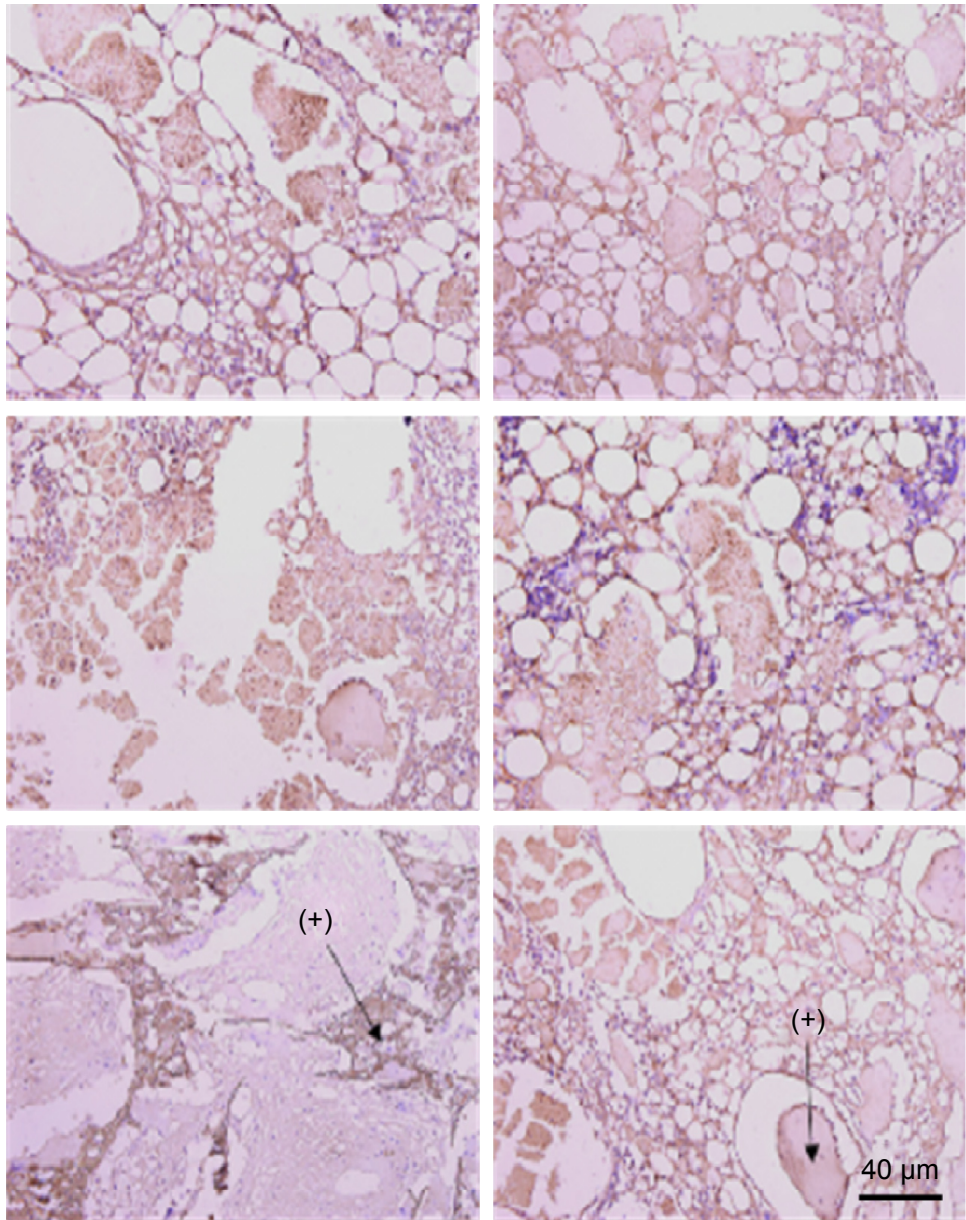

8 weeks

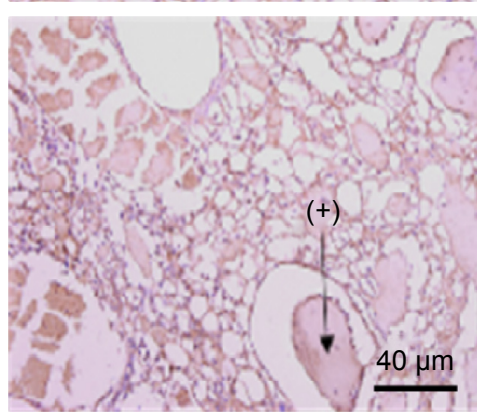

12 weeks

Figure 8 Immunohistological staining of VEGF by histological sections after CSD (A), 10 nl-MSC (B) and 20 nl-MSC (C) cements were implanted in vivo for 4, 8 and 12 weeks. Purple color and plus sign indicate positive expression of VEGF.

Abbreviations: VEGF, vascular endothelial growth factor; CSD, calcium sulfate dihydrate; nl-MSC, nanoporous lithium doping magnesium silicate composite.

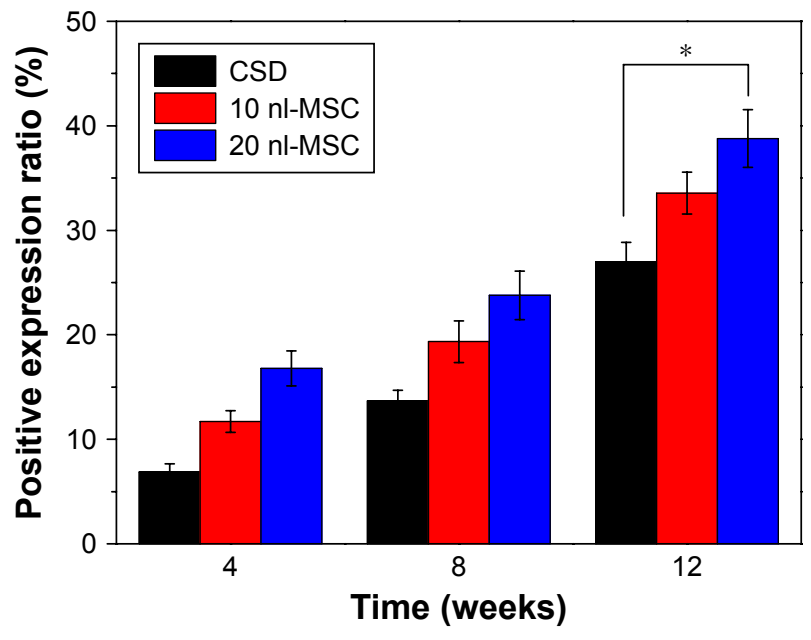

Figure 9 Quantitative analysis of the immunohistological positive expression of VEGF by histological sections after the implantation of cements in vivo for 4,8 and 12 weeks. Asterisk represents statistical significance $(P<0.05)$.

Abbreviations: VEGF, vascular endothelial growth factor; CSD, calcium sulfate dihydrate; nl-MSC, nanoporous lithium doping magnesium silicate composite.

\section{Discussion}

Calcium sulfate as a bone implant biomaterial has been clinically used for more than a century. However, many studies have clearly revealed that the shortage of calcium sulfate was not osteoinductive or osteogenic. ${ }^{15-17}$ In this study, nl-MS was synthesized, and the nl-MS-containing calcium sulfate bone cements were prepared. The result indicated that the addition of nl-MS increased the degradability of the nl-MSC cements, which depended on the nl-MS content. The CSD cement was a compact structure after the CSH transformed into CSD, while the $10 \mathrm{nl}-\mathrm{MSC}$ and $20 \mathrm{nl}-\mathrm{MSC}$ cements were loose microporous structures after addition of nl-MS into CSD. The nl-MS with nanoporosity distributed evenly into the cements, which undermined the compact structure of CSD cement. Moreover, the unique nanostructure properties of nl-MS, including high specific surface area and large pore volume, 
A

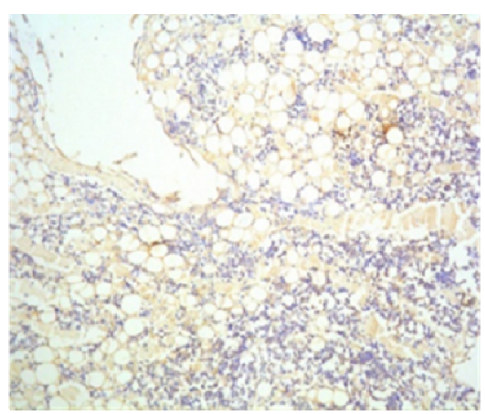

B

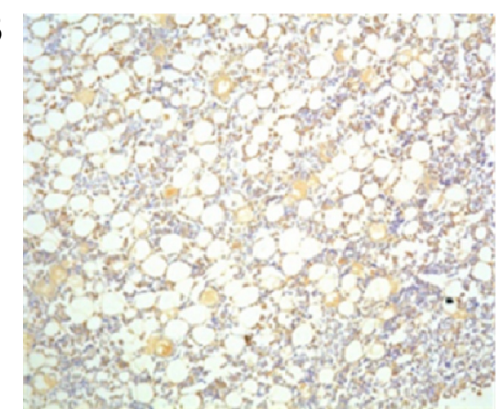

C

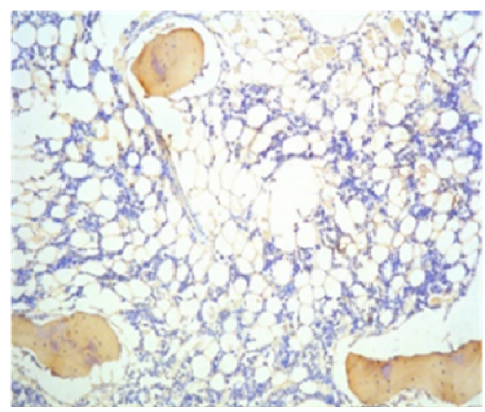

4 weeks
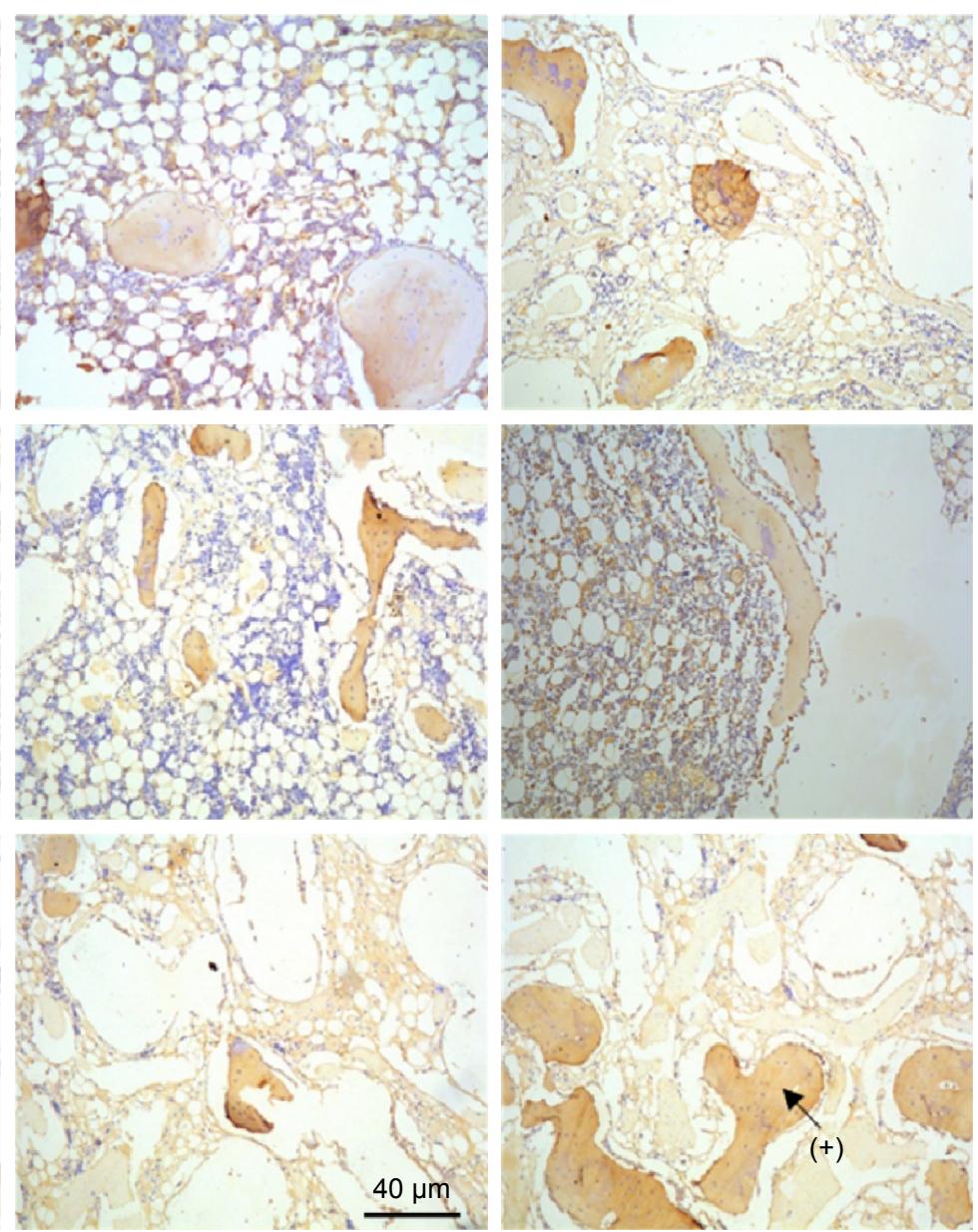

8 weeks

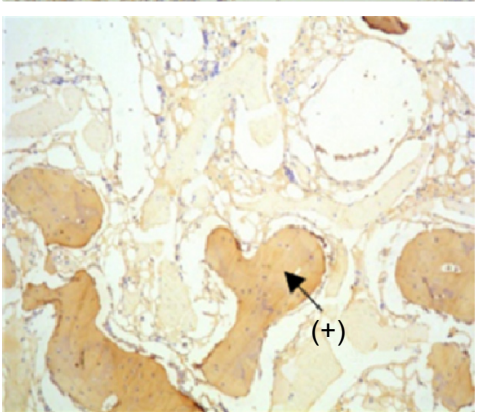

12 weeks

Figure 10 Immunohistological staining of COL-I by histological sections after CSD (A), 10 nl-MSC (B) and $20 \mathrm{nl}-\mathrm{MSC}$ (C) were implanted in vivo for 4, 8 and 12 weeks. Brown color and plus sign indicate positive expression of COL-I.

Abbreviations: COL-I, collagen type I; CSD, calcium sulfate dihydrate; nI-MSC, nanoporous lithium doping magnesium silicate composite.

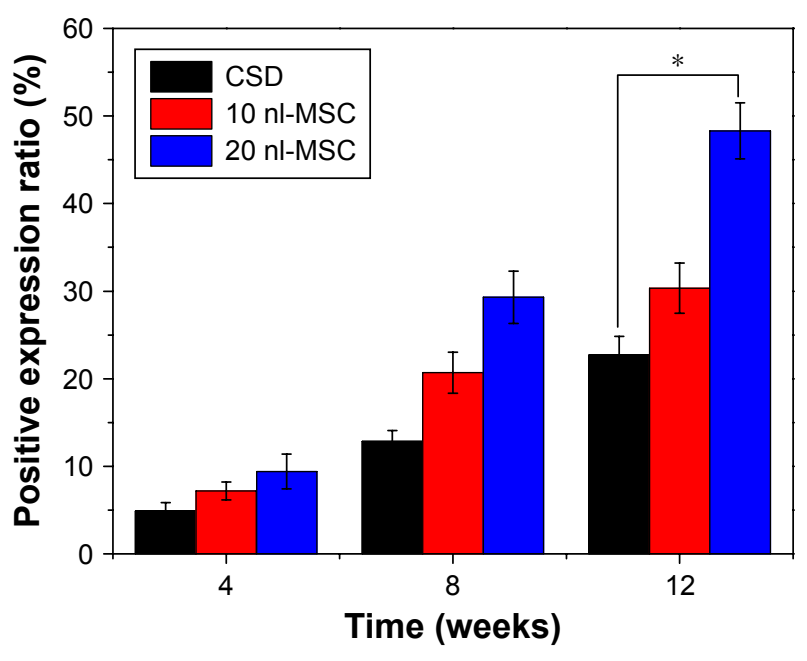

Figure II Quantitative analysis of the immunohistological positive expression of COL-I by histological sections after the implantation of cements in vivo for 4,8 and 12 weeks. Asterisk represents statistical significance $(P<0.05)$.

Abbreviations: COL-I, collagen type I; CSD, calcium sulfate dihydrate; nI-MSC, nanoporous lithium doping magnesium silicate composite. increased the porosity of the cements. Therefore, incorporation of nl-MS into CSD formed a microporous structure in the $10 \mathrm{nl}-\mathrm{MSC}$ and $20 \mathrm{nl}-\mathrm{MSC}$ cements, which increased the contact area with water when soaked into liquid, and thus improved the degradability of the nl-MSC cements.

Some studies have shown that the degradation of CSD produced acidic degradable products and formed a local acidic microenvironment, which caused inflammatory reaction in vivo. ${ }^{18,19}$ In this study, the $\mathrm{pH}$ of the solution decreased from 7.40 to 6.86 after the CSD cement immersion into PBS for 12 weeks, indicating that some acidic degradable products emerged during soaking. However, the $\mathrm{pH}$ of the solution for 20 nl-MSC slightly increased from 7.40 to 7.45 , indicating that some basic products emerged after the degradation of nl-MS, which might neutralize the acidic degradable products of CSD. According to previous studies, a slightly alkalinized microenvironment might be favorable for cell 
growth and tissue formation. ${ }^{20,21}$ Therefore, the $\mathrm{pH}$ of 7.45 for $20 \mathrm{~mL}-\mathrm{MSC}$ might promote cells/bone tissue ingrowth and new bone regeneration.

In order to investigate the in vivo degradability, vascularization and osteogenesis of the bone cements, the specimens were implanted into femur defects of rabbits. The results revealed that all the cements exhibited excellent biocompatibility, and neither significant foreign body response nor chronic inflammation to the cements was observed throughout the in vivo study. The results of the cross-section of the $3 \mathrm{D}$ reconstruction images of SRmCT revealed that the bone defects were gradually repaired with time after the cements were implanted into bone defects of rabbit femur for different times. The effects of bone defects repair increased with the time and nl-MS content, in which 20 nl-MSC was the best for defects repair at 12 weeks, indicating that the osteogenesis of $20 \mathrm{nl}-\mathrm{MSC}$ was the best. It can be suggested that the fast degradation of $20 \mathrm{nl}-\mathrm{MSC}$ and release of $\mathrm{Si}$ and $\mathrm{Li}$ ions promoted osteogenesis and repaired the bone defects. These results indicated that the addition of nl-MS into the cements promoted the bone defects repair. The results of the histological sections of H\&E staining showed that the amounts of new bone tissue increased not only with time but also with the increase of nl-MS content in the cements. The results indicated that the addition of nl-MS improved the new bone formation of nl-MSC cements. Moreover, the residual material ratio of the cements decreased with time, and with the increase of nl-MS content, indicating that addition of nl-MS improved the degradability of nl-MSC cements and new bone formation. The nl-MSC cements were degradable at skeletal sites over 12 weeks. Therefore, the degradation rate of nl-MSC cements can meet the requirement of bone defects repair. The first degradation of nl-MS in the nl-MSC cements at early stage led to microporous structure in the specimens, which was favorable for the new bone tissue formation and ingrowth. The degradation rate of nl-MSC increased slightly with the increase of nl-MS content in vivo, which was consistent with the in vitro degradation. It can be suggested that the nl-MSC cements not only had good biocompatibility and osteogenesis, but were also gradually degradable over a period of time and progressively replaced by the natural host bone tissue.

Insufficient vascularization would restrain new bone tissue formation and delay bone defects healing, possibly because of deviations from the principles of vasculature functioning in osteogenesis. ${ }^{22,23}$ Moreover, the vascular development always precedes the osteogenesis, and bone tissue ingrowth greatly depends on the vascularization. Under the circumstance of a well-developed vascular network, the osteoblasts produce osteoid, calcify and differentiate to osteocytes and exhibit healthy bone formation. ${ }^{24}$ VEGF has also been reported to stimulate the osteogenic differentiation of osteoblasts. ${ }^{25}$ In this study, the results from immunohistochemical analysis showed that the nl-MSC cements containing nl-MS significantly promoted the positive expression of VEGF, which was nl-MS content-dependent (CSD $<10$ nl-MSC $<20$ nl-MSC). Clearly, the nl-MS obviously enhanced the expression of VEGF, which stimulated the vascularization.

COL-I is a major component of extracellular matrix deposition, which accounts for $90 \%$ of the bone matrix proteins. ${ }^{26}$ COL-I is secreted at the early phase of osteoid matrix deposition, so it is an early marker of osteogenic differentiation associated with the formation of extracellular matrix..$^{27,28}$ In this study, the results from immunohistochemical analysis showed that the cements containing nl-MS significantly promoted the positive expression ratio of COL-I, which was nl-MS content-dependent (CSD $<10$ nl-MSC $<20$ nl-MSC), indicating that nl-MSC cements containing nl-MS promoted excellent in vivo osteogenesis for bone defects repair.

Previous studies revealed that silicate-based biomaterials with simple composition of Si stimulated osteogenic differentiation of BMSCs and angiogenesis of human umbilical vein endothelial cells. ${ }^{29}$ In the present study, VEGF and COL-I expressions for both $20 \mathrm{nl}-\mathrm{MSC}$ and $10 \mathrm{nl}-\mathrm{MSC}$ cements were significantly upregulated by addition of nl-MS, and it can be reasoned that the nl-MS played important roles in the upregulation of vascularization and osteogenic differentiation. Therefore, this study demonstrated that the nl-MSC cements containing nl-MS clearly enhanced the vascularization and osteogenic differentiation of cells as compared to the CSD. The results also proved the feasibility that the incorporation of proper amount of nl-MS into CSD could enhance the stimulatory effects on vascularization and osteogenic differentiation as compared to CSD.

Previous studies have shown that the $\mathrm{Si}$ and $\mathrm{Li}$ ions could promote the proliferation and differentiation of osteoblasts. ${ }^{14,30}$ In this study, the degradation of nl-MSC could lead to gradual release of $\mathrm{Si}$ and $\mathrm{Li}$ ions both in vitro and in vivo, which might be critically important for regulating various cellular processes of osteoblasts, including cell proliferation, differentiation and new bone tissue formation. Therefore, the better bone-regenerating ability of nl-MSC cements was due to fast degradation after addition of nl-MS and the release of $\mathrm{Si}$ and $\mathrm{Li}$ ions, and formed a $\mathrm{Li}$ - and $\mathrm{Si}$-rich microenvironment in vivo, which might show the synergistic effects on stimulating the proliferation and differentiation of 
osteoblasts, and then promoted osteogenesis and bone tissue regeneration.

\section{Conclusion}

In this study, degradable nl-MSC cements were prepared by adding nl-MS into $\mathrm{CSH}$. The results revealed that the incorporation of nl-MS into the cements obviously improved the degradability of nl-MSC cements both in vitro and in vivo. Moreover, the nl-MSC cements containing nl-MS showed the ability to neutralize the acidic degradation by-products released from the cements and prevented the $\mathrm{pH}$ from decreasing during degradation. The SRmCT and histological elevation results revealed that the nl-MSC cements containing nl-MS obviously enhanced new bone formation and promoted the degradability of the cements in vivo, indicating good biocompatibility. In addition, the immunohistochemical analysis results revealed that the nl-MSC cements containing nl-MS could promote the positive expression of VEGF and COL-I, indicating that nl-MSC could enhance the vascularization and osteogenic differentiation in vivo. In short, the nl-MSC cements with good biocompatibility and degradability could promote vascularization and osteogenesis, which have great potential to treat bone defects.

\section{Acknowledgments}

This study was supported by grants from the National Natural Science Foundation of China (31271031, 31100680), the Natural Science Fund of Shanghai (15ZR1412500), the Major International Joint Research Project between China and Korea (81461148033) and the National Research Foundation of Korea (NRF) (NRF-2014K2A2A7066637).

\section{Disclosure}

The authors report no conflicts of interest in this work.

\section{References}

1. Chen Z, Kang L, Meng QY, et al. Degradability of injectable calcium sulfate/mineralized collagen-based bone repair material and its effect on bone tissue regeneration. Mater Sci Eng C Mater Biol Appl. 2014;45: 94-102.

2. Liu W, Wu C, Liu W, Zhai W, Chang J. The effect of plaster $\left(\mathrm{CaSO}_{4} \cdot 1 / 2 \mathrm{H}_{2} \mathrm{O}\right)$ on the compressive strength, self-setting property, and in vitro bioactivity of silicate-based bone cement. J Biomed Mater Res B Appl Biomater. 2013;101(2):279-286.

3. Stubbs D, Deakin M, Chapman-Sheath $P$, et al. In vivo evaluation of resorbable bone graft substitutes in a rabbit tibial defect model. Biomaterials. 2004;25(20):5037-5044.

4. Chen CC, Wang CW, Hsueh NS, Ding SJ. Improvement of in vitro physicochemical properties and osteogenic activity of calcium sulfate cement for bone repair by dicalcium silicate. J Alloys Compd. 2014; 585:25-31.
5. Chen WL, Chen CK, Lee JW, Lee YL, Ju CP, Lin JH. Structure, properties and animal study of a calcium phosphate/calcium sulfate composite cement. Mater Sci Eng C Mater Biol Appl. 2014;37: 60-67.

6. Wu Z, Tang T, Guo H, et al. In vitro degradability, bioactivity and cell responses to mesoporous magnesium silicate for the induction of bone regeneration. Colloids Surf B Biointerfaces. 2014;120:38-46.

7. Arcos D, Vallet-Regí M. Sol-gel silica-based biomaterials and bone tissue regeneration. Acta Biomater. 2010;6(8):2874-2888.

8. Guo Z, Liu XM, Ma L, et al. Effects of particle morphology, pore size and surface coating of mesoporous silica on Naproxen dissolution rate enhancement. Colloids Surf B Biointerfaces. 2013;101:228-235.

9. Lin KL, Liu Y, Huang H, Chen L, Wang Z, Chang J. Degradation and silicon excretion of the calcium silicate bioactive ceramics during bone regeneration using rabbit femur defect model. J Mater Sci Mater Med. 2015;26(6):197.

10. Khan AF, Saleem M, Afzal A, Ali A, Khan A, Khan AR. Bioactive behavior of silicon substituted calcium phosphate based bioceramics for bone regeneration. Mater Sci Eng C Mater Biol Appl. 2014;35: $245-252$.

11. Zhou H, Wu X, Wei J, et al. Stimulated osteoblastic proliferation by mesoporous silica xerogel with high specific surface area. J Mater Sci Mater Med. 2011;22(3):731-739.

12. Han P, Wu C, Chang J, Xiao Y. The cementogenic differentiation of periodontal ligament cells via the activation of $\mathrm{Wnt} / \beta$-catenin signalling pathway by $\mathrm{Li}+$ ions released from bioactive scaffolds. Biomaterials. 2012;33(27):6370-6379.

13. Barry F, Murphy M. Mesenchymal stem cells in joint disease and repair. Nat Rev Rheumatol. 2013;9(10):584-594.

14. Wu Y, Zhu S, Wu C, et al. A bi-lineage conducive scaffold for osteochondral defect regeneration. Adv Funct Mater. 2014;24(28): 4473-4483.

15. Zhao J, Shen G, Liu C, et al. Enhanced healing of rat calvarial defects with sulfated chitosan-coated calcium-deficient hydroxyapatite/ bone morphogenetic protein 2 scaffolds. Tissue Eng Part A. 2012; 18(1-2):185-197.

16. Chen Z, Liu H, Liu X, et al. Improved workability of injectable calcium sulfate bone cement by regulation of self-setting properties. Mater Sci Eng C Mater Biol Appl. 2013;33(3):1048-1053.

17. He X, Dziak R, Mao K, et al. Integration of a novel injectable nano calcium sulfate/alginate scaffold and BMP2 gene-modified mesenchymal stem cells for bone regeneration. Tissue Eng Part A. 2013;19(3-4): 508-518.

18. Walsh WR, Morberg P, Yu Y, et al. Response of a calcium sulfate bone graft substitute in a confined cancellous defect. Clin Orthop Relat Res. 2003;(406):228-236.

19. Thomas MV, Puleo DA. Calcium sulfate: properties and clinical applications. J Biomed Mater Res B Appl Biomater. 2009;88(2):597-610.

20. Cheng N, Wang Y, Zhang Y, Shi B. The osteogenic potential of mesoporous bioglasses/silk and non-mesoporous bioglasses/silk scaffolds in ovariectomized rats: in vitro and in vivo evaluation. PLoS One. 2013; 8(11):e81014.

21. Foppiano S, Marshall SJ, Marshall GW, Saiz E, Tomsia AP. The influence of novel bioactive glasses on in vitro osteoblast behavior. J Biomed Mater Res A. 2004;71(2):242-249.

22. Kulanthaivel S, Mishra U, Agarwal T, et al. Improving the osteogenic and angiogenic properties of synthetic hydroxyapatite by dual doping of bivalent cobalt and magnesium ion. Ceram Int. 2015;41(9): 11323-11333.

23. Li H, Xue K, Kong N, Liu K, Chang J. Silicate bioceramics enhanced vascularization and osteogenesis through stimulating interactions between endothelia cells and bone marrow stromal cells. Biomaterials. 2014; 35(12):3803-3818.

24. Jha AK, Tharp KM, Browne S, et al. Matrix metalloproteinase-13 mediated degradation of hyaluronic acid-based matrices orchestrates stem cell engraftment through vascular integration. Biomaterials. 2016; 89:136-147. 
25. Zhang $\mathrm{W}$, Zhu $\mathrm{C}, \mathrm{Wu} \mathrm{Y}$, et al. VEGF and BMP-2 promote bone regeneration by facilitating bone marrow stem cell homing and differentiation. Eur Cell Mater. 2014;27:1-11; discussion 11-12.

26. Cheng S, Wang W, Lin Z, et al. Effects of extracellular calcium on viability and osteogenic differentiation of bone marrow stromal cells in vitro. Hum Cell. 2013;26(3):114-120.

27. Zhang Y, Li S, Wu C. The in vitro and in vivo cementogenesis of $\mathrm{CaMgSi}_{2} \mathrm{O}_{6}$ bioceramic scaffolds. J Biomed Mater Res A. 2014;102(1): $105-116$.

28. Reffitt DM, Ogston N, Jugdaohsingh R, et al. Orthosilicic acid stimulates collagen type 1 synthesis and osteoblastic differentiation in human osteoblast-like cells in vitro. Bone. 2003;32(2):127-135.
29. Shie MY, Chiang WH, Chen IP, Liu WY, Chen YW. Synergistic acceleration in the osteogenic and angiogenic differentiation of human mesenchymal stem cells by calcium silicate-graphene composites. Mater Sci Eng C. 2017;73:726-735.

30. Sun J, Wei L, Liu X, et al. Influences of ionic dissolution products of dicalcium silicate coating on osteoblastic proliferation, differentiation and gene expression. Acta Biomaterialia. 2009;5(4):1284-1293.

\section{Publish your work in this journal}

The International Journal of Nanomedicine is an international, peerreviewed journal focusing on the application of nanotechnology in diagnostics, therapeutics, and drug delivery systems throughout the biomedical field. This journal is indexed on PubMed Central, MedLine, CAS, SciSearch ${ }^{\circledR}$, Current Contents ${ }^{\circledR} /$ Clinical Medicine,
Journal Citation Reports/Science Edition, EMBase, Scopus and the Elsevier Bibliographic databases. The manuscript management system is completely online and includes a very quick and fair peer-review system, which is all easy to use. Visit http://www.dovepress.com/ testimonials.php to read real quotes from published authors. 